\title{
Morbilidade Neonatal na Diabetes Gestacional: Coincidência ou Consequência do Consenso de 2011
}

\section{Neonatal Morbidity and Gestational Diabetes: Coincidence or Consequence of the 2011 Protocol}

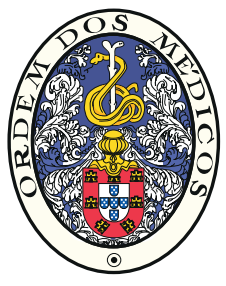

\author{
Gabriela MIMOSO $\triangle^{1}$, Guiomar OLIVEIRA ${ }^{2,3}$ \\ Acta Med Port 2017 Sep;30(9):589-598 - https://doi.org/10.20344/amp.8033
}

\section{RESUMO}

Introdução: A diabetes gestacional constitui uma das doenças associada à gravidez com maior taxa de complicações. Apesar de ser uma condição habitualmente transitória, estão reconhecidas as suas complicações a curto e longo prazo. Há evidência científica para afirmar que um bom controlo metabólico reduz as complicações perinatais. Em 2011, foram propostos novos critérios para o seu diagnóstico, o que o tornou possível logo no primeiro trimestre de gravidez. Neste trabalho, propomo-nos comparar a morbilidade neonatal entre dois grupos de recém nascidos filhos de mulheres com diabetes gestacional submetidos a dois protocolos diferentes.

Material e Métodos: Estudo observacional analítico, retrospetivo descritivo de recém nascidos de mães com diabetes gestacional com gravidez unifetal seguida na Maternidade Bissaya Barreto no período de 2008 a 2013. Utilizou-se a informação clinica de recém nascidos e mães com diabetes gestacional armazenada em base de dados. Analisaram-se as repercussões clínicas da utilização do novo consenso comparando dois períodos de três anos.

Resultados: Analisaram-se 774 díades mãe-filho. No segundo período a diabetes gestacional foi diagnosticada mais precocemente e a terapêutica com insulina foi instituída com mais frequência. Registou-se um aumento significativo da morbilidade neonatal, com mais casos de hipoglicémia neonatal e de anomalias congénitas e maior taxa de recém nascidos leves para a idade gestacional.

Discussão: O aumento da morbilidade neonatal, nos últimos anos, associou-se de um modo positivo à precocidade do diagnostico de diabetes gestacional e ao rigor do controlo metabólico.

Conclusão: Analisar os dados nacionais será fundamental para compreender este inesperado e preocupante aumento da morbilidade. Palavras-chave: Complicações na Gravidez; Diabetes Gestacional; Doenças do Recém-Nascido

\section{ABSTRACT}

Introduction: Gestational diabetes is one of the diseases associated with pregnancy with higher rate of complications. Despite being a transitory condition, short and long term complications related to gestational diabetes have been described. There is scientific evidence to say that good metabolic control decreases perinatal complications. In 2011, new criteria was proposed for its diagnosis, which made possible its diagnosis during the $1^{\text {st }}$ trimester of pregnancy. The aim of this study is to compare neonatal morbidity in two groups of women with gestational diabetes diagnosis before and after the latest Portuguese guidelines for diabetes and pregnancy were published (February 2011).

Material and Methods: We included all newborns born in Maternidade Bissaya Barreto whose mother, followed at our maternity between 2008 and 2013, had unifetal pregnancy complicated by diabetes. We used a perinatal database and analysed the impact of the new guidelines in perinatal morbidity over two periods of three years.

Results: There were 774 women who met the inclusion criteria. We found that gestational diabetes was diagnosed earlier, insulin therapy was more frequent. Neonatal morbidity was increased, and there were more cases of neonatal hypoglycemia and congenital anomalies, and newborns became smaller for gestational age.

Discussion: The increase in neonatal morbidity was associated with early diagnosis and rigorous metabolic control.

Conclusion: To analyse national data will be fundamental to understand this unexpected increase in morbidity.

Keywords: Diabetes, Gestational; Infant, Newborn, Diseases; Pregnancy Complications

\section{INTRODUÇÃO}

A diabetes gestacional (DG) é uma intolerância à glicose que se desencadeia com a gravidez, de gravidade variável, podendo apresentar-se desde formas ligeiras de intolerância até à diabetes prévia à gravidez, ainda não identificada. ${ }^{1-3}$

A DG é atualmente diagnosticada pela determinação da glicémia em jejum em consulta pré-natal. Se este valor for normal realiza-se a prova de tolerância à glicose oral (PTGO) com a administração de $75 \mathrm{~g}$ de glicose entre as 24 e as 28 semanas de gestação. A maioria dos casos $(70 \%$ a $85 \%$ ) de DG é adequadamente tratada com dieta e exer-

cício físico, contudo, algumas grávidas necessitam de terapêutica com insulina. ${ }^{3-8}$

O rastreio universal de DG vigora em Portugal ${ }^{9}$ sendo sustentado pelo facto de $30 \%$ a $50 \%$ das mulheres não apresentarem fatores de risco para esta condição. $8,10,11$

Esta forma de diagnosticar a DG é suportada por estudos clínicos que, como o Hyperglycemia and Adverse Pregnancy Outcomes (HAPO), ${ }^{12}$ demonstraram uma associação linear positiva entre o valor da glicémia e as complicações da gravidez em mulheres sem DG. 2,5,6,9,11-15

A DG é considerada uma das complicações da gravidez

1. Serviço de Neonatologia. Maternidade Bissaya Barreto. Centro Hospitalar e Universitário de Coimbra. Coimbra. Portugal.

2. Departamento de Pediatria. Faculdade de Medicina. Universidade de Coimbra. Coimbra. Portugal.

3. Unidade de Neurodesenvolvimento e Autismo do Centro de Desenvolvimento da Criança e Centro de Investigação e Formação Clínica. Hospital Pediátrico de Coimbra. Centro Hospitalar e Universitário de Coimbra. Coimbra. Portugal.

$\square$ Autor correspondente: Gabriela Mimoso.gabriela.mimoso@gmail.com

Recebido: 11 de julho de 2016 - Aceite: 12 de abril de 2017 | Copyright @ Ordem dos Médicos 2017 
associada a maior morbilidade perinatal, ${ }^{5,6,11,13,16}$ sobretudo se não for adequadamente tratada. Nos recém-nascidos (RN) destacam-se pela sua frequência a hipoglicémia neonatal, o síndroma de dificuldade respiratória, a prematuridade e a hiperbilirrubinémia ${ }^{3,8,17-19}$ e na mãe a hipertensão

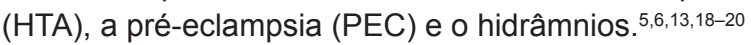

Para além da morbilidade a curto prazo, está bem documentada a sua associação, a médio e longo prazo, a diabetes e síndroma metabólico na mãe e no filho a obesidade, alterações do metabolismo da glicose e doença cardiovascular. ${ }^{9,16,21,22}$

A prevalência da DG varia de acordo com a população estudada e os critérios utilizados. ${ }^{16,22,23} \mathrm{Em}$ Portugal em 2013 foi estimada em $5,8 \%,{ }^{24} 1,7$ vezes superior à do período entre 2007 e $2008 .^{7,25}$

O objetivo principal deste trabalho foi o de analisar de que forma o novo protocolo de diagnóstico e abordagem da DG influenciou a morbimortalidade perinatal.

\section{MATERIAL E MÉTODOS}

Estudo observacional analítico com análise retrospetiva de dados armazenados numa base clínica digital de uma população de grávidas com diabetes estudadas e seguidas na MBB, durante o período de 2008 a 2013 (seis anos).

Critérios de inclusão: gravidez unifetal com diagnóstico de DG vigiada e com parto na MBB. O diagnóstico foi confirmado nos três primeiros anos, com dois passos, mediante a realização de uma prova de rastreio (teste de O'Sullivan - administração de $50 \mathrm{~g}$ de glicose oral com determinação da glicémia 1 hora depois) entre as 24 e as 28 semanas de gestação, seguido da prova diagnóstica (prova de tolerância à glicose oral - PTGO) com ingestão de 100 $\mathrm{g}$ de glicose. Após fevereiro de 2011 o diagnóstico tem sido efetuado apenas num passo, com a determinação da glicémia em jejum no primeiro trimestre de gravidez ou com a PTGO entre as 24 e as 28 semanas.

De acordo com as recomendações da Organização Mundial de Saúde, todas as mulheres com DG deverão realizar uma PTGO com $75 \mathrm{~g}$ de glicose, seis a oito semanas após o parto, para reclassificação. ${ }^{9}$

Foram analisadas as seguintes variáveis das mães: idade, antecedentes pessoais de DG, peso e IMC prévio à gravidez atual. Na evolução da gravidez foram avaliados: fatores de risco para a ocorrência da DG (história familiar de diabetes, antecedentes de diabetes gestacional, idade superior a 35 anos, multiparidade e história prévia de macrossomia, abortos de repetição, malformação fetal ou morte fetal intrauterina - MFIU), IG no momento do diagnóstico, ganho ponderal na gravidez de acordo com os valores padrão definidos pelo Institute of Medicine (IM) 2009, ${ }^{26}$ taxa de insulinização e IG do seu início e complicações obstétricas. Relativamente ao RN estudou-se: IG e via do parto, peso ao nascimento $(P N)$, género, morbilidade (necessidade de reanimação, trauma de parto, anomalias congénitas e complicações metabólicas) e mortalidade perinatal.

Na mãe foram usadas as seguintes definições:

Patologia na gravidez, considerou-se para o efeito qual- quer outro evento que perturbe o decorrer da gravidez, para além da diabetes gestacional: hipertensão, PEC, infeção urinária (IU), colestase, ameaça de parto prematuro (APP), rotura prematura de membranas (RPM).

A mulher considerou-se como tendo peso excessivo ou sendo obesa quando o IMC prévio à gravidez foi superior a $25 \mathrm{~kg} / \mathrm{m}^{2}$ e $30 \mathrm{~kg} / \mathrm{m}^{2}$, respetivamente. A terapêutica com insulina foi instituida de acordo com as recomendações do consenso nacional de diabetes e gravidez. ${ }^{9}$

O PN foi quantificado nos primeiros minutos de vida numa balança Seca(R), por enfermeiras qualificadas. Adotaram-se as curvas de Olsen ${ }^{27}$ para classificação dos RN na relação entre o percentil $(P)$ do $P N$ e a IG, consideraram-se: leves para a idade gestacional (LIG) - PN < P10, adequados para a idade gestacional (AIG) - PN: P10 - 90; e grandes para a idade gestacional (GIG), PN > P90.

No RN foram usadas as seguintes definições:

- Hipoglicémia: glicémia capilar inferior a 40 mg/dL (2.2 $\mathrm{mmol} / \mathrm{L}$ ) em qualquer momento da estadia hospitalar, independentemente da IG e da idade pós-natal. A hipoglicémia num RN assintomático conduziu a reforço no apoio à amamentação e/ou administração oral de um soluto de glicose a $10 \%(2 \mathrm{ml} / \mathrm{kg})$ com repetição da glicémia capilar cerca de uma hora depois. Os RN com hipoglicémia sintomática ou assintomática persistente são internados em unidade de cuidados intensivos neonatais (UCIN) para monitorização clínica e perfusão de glicose a $10 \%$.

- Hiperbilirrubinémia (quantificada por química seca): Icterícia com critérios de fototerapia com base no protocolo em uso na $\mathrm{MBB}^{28}$ que tem em consideração a IG e as horas de vida. Foram excluídos os casos de icterícia patológica.

- Encefalopatia hipóxico-isquémica (EHI): Quadro clínico com sintomatologia neurológica (hipotonia, ausência de sucção, irritabilidade, convulsões ou coma) na sequência de um insulto asfíxico no momento do parto (Índice de Apgar (IA), inferior a sete ao quinto minuto e necessidade de reanimação) e que se traduziu por acidose metabólica $(\mathrm{pH}<7.00, \mathrm{BE} \leq-16 \mathrm{mmol} / \mathrm{L}$ na primeira hora de vida) e alterações na ecografia cerebral.

- Hipocalcémia (quantificada por química seca): Cálcio sérico inferior a $2 \mathrm{mmol} / \mathrm{L}$.

- Morbilidade composta ou combinada: Presença de uma das seguintes morbilidades: IA ao quinto minuto inferior a sete, EHI, convulsões, hipoglicemia, hipocalcémia, hiperbilirrubinémia, policitémia, dificuldade respiratória, paralisia de Erb ou fratura da clavícula ou necessidade de internamento em cuidados intensivos ou intermédios (por motivos médicos).

A morbimortalidade perinatal desta população foi comparada com a dos outros RN da MBB durante o período em análise. Utilizou-se para o efeito os códigos de diagnóstico da classificação internacional de doenças, nona edição (ICD9) para indexação dos registos hospitalares, classificados por um neonatologista do serviço. Os dados foram obtidos através do programa de auditoria clínica dos grupos de diagnósticos homogéneos (GDH).

Para a análise estatística utilizou-se o programa 
Statistical Package for the Social Sciences - SPSS versão 20. Considerou-se um nível de significância de 0,05. Procedeu-se à análise bivariada para identificar diferenças entre grupos; utilizou-se o teste de Mann-Whitney para comparação de variáveis contínuas e o teste de qui-quadrado/ teste de Fisher para comparação de variáveis categóricas. Calcularam-se os odds ratio (OR) e os odds ratio ajustados (ORa) após regressão logística binária das variáveis estatisticamente significativas.

Critérios de exclusão: Foram excluídas as gravidezes gemelares e aquelas em que a vigilância ou o parto não ocorreram na MBB.

\section{RESULTADOS}

No período considerado nasceram na MBB 17211 recém-nascidos. A gravidez complicou-se com DG em 970. Após aplicação dos critérios de exclusão, estudaram-se 774 díades mães e RN, distribuídos pelos dois períodos de estudos. Obtiveram-se 421 RN no período entre 2008 a 2010 que se compararam com os 353 RN nascidos entre 2011 e 13.

Nos últimos três anos (Tabela 1), o número de grávidas com fatores de risco para DG diminuiu mas sem significado estatístico e destacando-se apenas a história de filho anterior com malformação que aumentou de $0,7 \%$ para $3,7 \%$. No período mais recente, salienta-se o maior número de mulheres com a glicémia em jejum alterada e a maior necessidade de insulinoterapia. Verificou-se uma taxa superior de APP e de RPM. Constatou-se um aumento não significativo da prematuridade e do número de cesarianas.

Globalmente podemos afirmar que o RN do período mais recente - grupo 2 (Tabela 1 ) teve uma morbilidade combinada mais elevada sobretudo à custa do aumento significativo do número de casos de hipoglicémia neonatal. Registou-se também um aumento não significativo das situações de dificuldade respiratória e hiperbilirrubinémia.

Tabela 1 - Morbilidade e mortalidade perinatal: mãe e filho

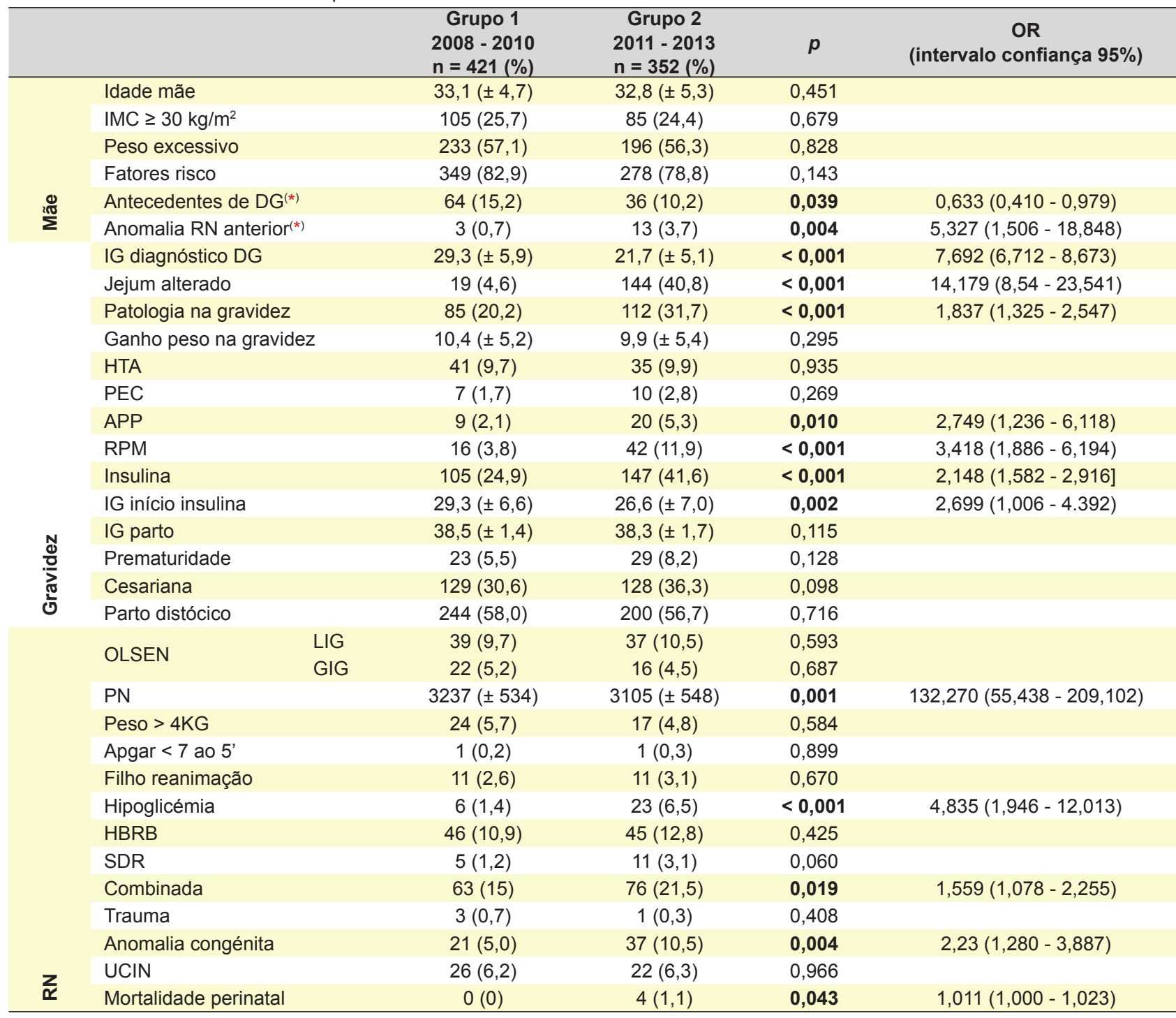

(*)-Excluídas as grávidas nulíparas; OR: odds ratio rotura de membranas; LIG: leve para a idade gestacional; GIG: grande para a idade gestacional (de acordo com as curvas de OLSEN); PN: peso nascimento; HBRB: hiperbilirrubinémia; SDR: síndroma de dificuldade respiratória; UCIN: unidade de Cuidados Intensivos Neonatais 
A prematuridade apesar de ter maior expressão (8,2\%) não aumentou significativamente com a mudança do protocolo de atuação (grupo 2) e verificou-se um aumento significativo do número total de anomalias congénitas no grupo 2 $(10,5 \%)$, sendo metade destas consideradas anomalias major.

Tabela 2 - Estudo das variáveis associadas à hipoglicémia
A hipoglicémia (Tabela 2 e Fig. 1) associou-se sobretudo a diagnóstico de DG mais precoce (antes das 10 semanas em $24,1 \%$ das mães), a existência de valor de jejum alterado e a gravidez complicada de HTA. Apesar de mais frequente em recém-nascidos prematuros, LIG e GIG tal associação não foi estatisticamente significativa.

\begin{tabular}{|c|c|c|c|c|c|c|}
\hline & & HIPOGLICÉM & & & & \\
\hline & $(\%)$ & $\underset{n=29}{\operatorname{Sim}}$ & $\begin{array}{c}\text { Não } \\
n=744\end{array}$ & $p$ & OR & $O R_{a j}$ \\
\hline & Idade materna (anos) & $32,3( \pm 6,2)$ & $33,1( \pm 4,9)$ & 0,548 & & \\
\hline & IMC $\left(\mathrm{kg} / \mathrm{m}^{2}\right)$ & $28,1( \pm 6,6)$ & $27( \pm 5,3)$ & 0,381 & & \\
\hline שֶ & $\mathrm{IMC}>30 \mathrm{~kg} / \mathrm{m}^{2}$ & $8(27,6)$ & $182(25,1)$ & 0,759 & & \\
\hline$\Sigma$ & Alt metabolismo glicose & $2(6,9)$ & $38(5,1)$ & 0,670 & & \\
\hline & IG diagnóstico (semanas) & $21,3( \pm 9,2)$ & $26,0( \pm 7,6)$ & 0,011 & $1,737(1,160-8,260)$ & $0,833(0,800-0,868)$ \\
\hline & Diagnóstico $<10 \mathrm{~s}$ & $7(24,1)$ & $65(8,7)$ & 0,005 & $3,324(1,368-8,076)$ & $0,097(0,030-0,321)$ \\
\hline & Jejum alterado & $13(44,8)$ & $149(20,3)$ & 0,002 & $3,185(1,499-6.766)$ & $6,353(3,498-11,539)$ \\
\hline & Insulina & $11(37,9)$ & $241(32,4)$ & 0,532 & & \\
\hline & IG inicio insulina (semanas) & $27( \pm 7,8)$ & $27,8( \pm 6,9)$ & 0,747 & & \\
\hline ט & Insulina $<10 \mathrm{~s}$ & $0(0)$ & $5(0,7)$ & 0,658 & & \\
\hline 口 & $\mathrm{HbA} 1 \mathrm{C}(\mathrm{mmol} / \mathrm{mol})$ & $5,2( \pm 0,53)$ & $5,2( \pm 0,57)$ & 0,752 & & \\
\hline & Ganho peso insuficiente & $11(37,9)$ & $261(35,1)$ & 0,753 & & \\
\hline & Ganho peso exagerado & $8(27,6)$ & $184(25,0)$ & 0,753 & & \\
\hline & HTA & $6(20,7)$ & $70(9,4)$ & 0,045 & $2,512(0,989-6,376)$ & \\
\hline & Cesariana & $11(37,9)$ & $246(33,1)$ & 0,585 & & \\
\hline$\frac{0}{0}$ & Prematuridade & $4(13,8)$ & $47(6,3)$ & 0,112 & & \\
\hline त্য & IG parto (semanas) & $37,7( \pm 2,2)$ & $38,4( \pm 1,5)$ & 0,076 & & \\
\hline & $I G<35 s$ & $2(6,9)$ & $13(1,7)$ & 0,049 & $4,165(0,895-19,380)$ & \\
\hline & Peso nascimento $(\mathrm{g})$ & $2968( \pm 644)$ & $3188( \pm 534)$ & 0,080 & & \\
\hline & Peso nascimento $>4 \mathrm{~kg}$ & $1(3,4)$ & $40(5,4)$ & 0,649 & & \\
\hline & LIG & $3(10,3)$ & $73(9,8)$ & 0,893 & & \\
\hline & GIG & $2(6,9)$ & $36(4,8)$ & 0,608 & & \\
\hline & Necessidade reanimação & $0(0)$ & $22(3,0)$ & 0,347 & & \\
\hline & UCIN & $6(20,7)$ & $42(5,6)$ & 0,001 & $4,360(1,685-11,284)$ & \\
\hline & SDR & $3(10,3)$ & $13(1,7)$ & 0,001 & $6,488(1,742-24,165)$ & \\
\hline & Clinica SNC & $2(6,9)$ & $3(0,4)$ & $<0,001$ & $18,296(2,935-114,050)$ & \\
\hline$z$ & Anomalia congénita & $3(10,3)$ & $55(7,4)$ & 0,554 & & \\
\hline$\underline{\Lambda}$ & Mortalidade & $1(3,4)$ & $2(0,3)$ & 0,109 & & \\
\hline
\end{tabular}

IMC: índice massa corporal; IG: idade gestacional; s: semana; HTA: hipertensão; LIG: leve para a idade gestacional; GIG: grande para a idade gestacional (de acordo com as curvas de OLSEN); UCIN: unidade de Cuidados Intensivos Neonatais; SNC: sistema nervoso central; OR: odds ratio; ORaj: odds ratio ajustado

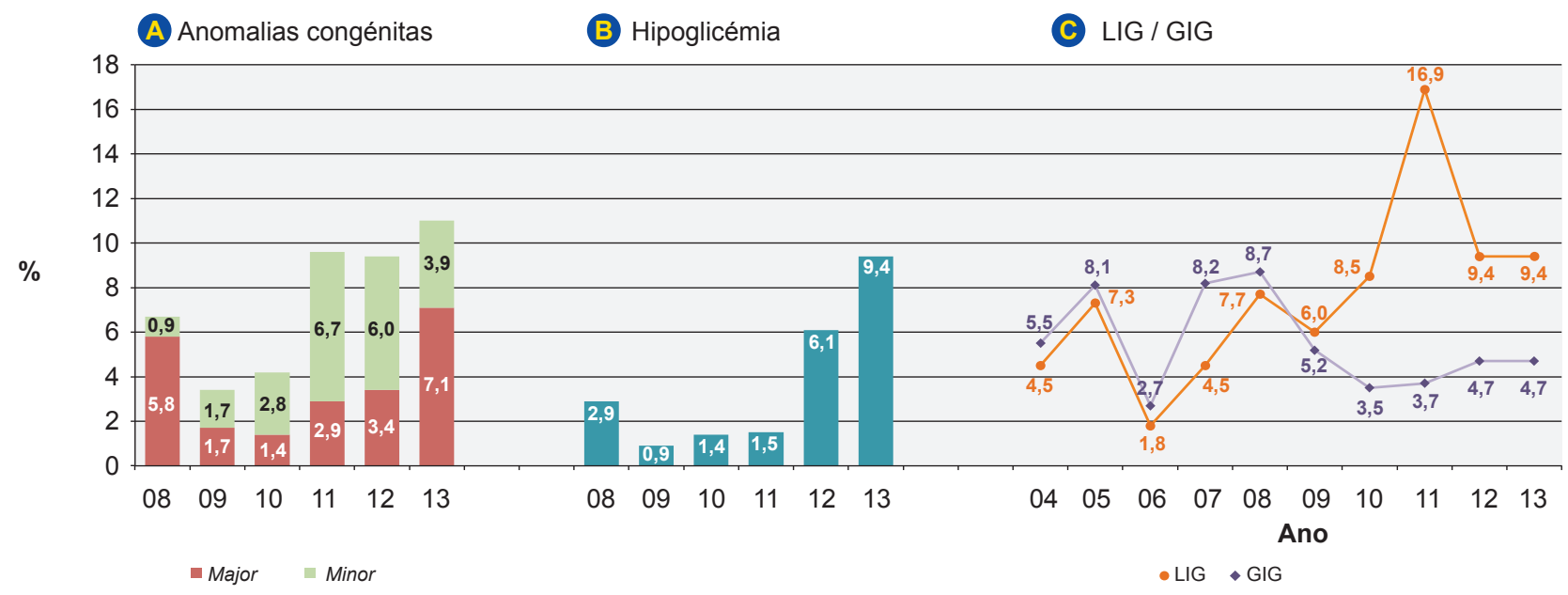

Figura 1 - Morbilidade neonatal mais frequente ao longo dos anos LIG: leva para a idade gestacional; GIG: grande para a idade gestacional 
A hipoglicémia ocorreu mais frequentemente em recém-nascidos internados na UCIN, com SDR e com clínica de patologia neurológica. Após análise de regressão logística, mantém significado estatístico para a ocorrência de hipoglicémia a IG no momento de diagnóstico da DG, sobretudo se diagnosticada antes das dez semanas e a existência do valor de jejum alterado.

As anomalias congénitas - Tabela 3 - foram mais frequentes em RN de mulheres com o diagnóstico de DG antes das dez semanas e submetidas a insulinoterapia, sobretudo quando esta foi introduzida precocemente (antes das dez semanas). As anomalias congénitas justificaram o internamento na UCIN num número significativo de RN $(17,2 \%$ vs $5,3 \%)$. As anomalias mais frequentes foram as cardiopatias $(n=10)$ e as esqueléticas $(n=8)$ seguidas das nefro-urológicas. Analisando o seu perfil evolutivo ao longo dos anos (Fig. 1) verificamos que a sua taxa é variável mas que existe uma tendência de aumento nos últimos três anos. Após análise de regressão logística, mantêm significado estatístico com OR ajustado $\left(\mathrm{OR}_{\mathrm{aj}}\right)$ para a ocorrência de anomalias congénitas a necessidade de insulina, sobretudo quando iniciada antes das dez semanas de IG.

Relativamente ao estado nutricional dos RN ao nascer observou-se uma tendência para a diminuição da macrossomia e dos GIG e para um aumento dos LIG (Fig. 1). Apesar da diminuição dos RN GIG (e dos macrossómicos) e explorando as variáveis a eles associados (Tabela 4) verificou-se que a sua ocorrência se associou fortemente à obesidade materna - IMC médio 31,1 vs $26,8 \mathrm{~kg} / \mathrm{m}^{2}$, à multiparidade, à história prévia de recém-nascidos GIG e à necessidade de insulinoterapia. $\mathrm{O}$ ganho excessivo de peso durante a gravidez foi decisivo para o filho ser GIG e em virtude disso o seu parto foi mais vezes distócico, na maior parte dos casos por cesariana. Se o ganho de peso materno excessivo foi determinante para se nascer GIG pelo contrário o insuficiente ganho ponderal associou-se a RN LIG (Tabela 4). Após análise de regressão logística, mantém significado estatístico para o RN ser GIG - o IMC prévio à gravidez, a existência de um filho anterior macrossómico e o ganho excessivo de peso na gravidez; pelo contrário nascer LIG manteve significado estatístico com o insuficiente aumento ponderal a que se associou a HTA gestacional.

Apesar do aumento da morbilidade o número de internamentos na UCIN manteve-se estável (cerca de 6\%) sendo os seus principais motivos a prematuridade, o SDR ( $1,1 \%$ com necessidade de ventilação), as anomalias congénitas e a hiperbilirrubinémia com necessidade de fototerapia intensiva. De salientar o número de internamentos associado a patologia infeciosa, vindo a falecer dois bebés (infeção neonatal a Streptococcus do grupo B e a Escherichia coli) sendo que em nenhum havia sido identificada patologia infeciosa na mãe (rastreio vaginal e na urocultura). Apenas no grupo 2 se registaram mortes neonatais, os dois já mencionados e uma situação de encefalopatia hipóxico-isquémica com necessidade de hipotermia. Neste período registou-se ainda uma MFIU às 28 semanas de gestação, atribuída a um acidente do cordão.

Tabela 3 - Estudo das variáveis associadas às anomalias congénitas

\begin{tabular}{|c|c|c|c|c|c|c|}
\hline & & \multicolumn{2}{|c|}{ ANOMALIA CONGÉNITA } & \multirow[b]{2}{*}{$p$} & \multirow[b]{2}{*}{ OR } & \multirow[b]{2}{*}{$\mathrm{OR}_{\mathrm{aj}}$} \\
\hline & & $\underset{n=58}{\operatorname{Sim}}$ & $\begin{array}{c}\text { Não } \\
n=716\end{array}$ & & & \\
\hline \multirow{7}{*}{$\sum^{2 \pi 0}$} & Idade materna (anos) & $33,4( \pm 5,1)$ & $33( \pm 4,5)$ & 0,601 & & \\
\hline & Idade $>35$ anos & $23(39,7)$ & $268(37,4)$ & 0,737 & & \\
\hline & IMC $\left(\mathrm{kg} / \mathrm{m}^{2}\right)$ & $26,8( \pm 5,9)$ & $27,1( \pm 5,4)$ & 0,785 & & \\
\hline & $\mathrm{IMC}>30 \mathrm{~kg} / \mathrm{m}^{2}$ & $8(14,8)$ & $182(25,9)$ & 0,070 & & \\
\hline & Malformação filho anterior & $2(3,4)$ & $14(2,0)$ & 0,442 & & \\
\hline & MFIU anterior & $1(1,7)$ & $9(1,3)$ & 0,762 & & \\
\hline & Alt metabolismo glicose & $2(3,4)$ & $38(5,3)$ & 0,761 & & \\
\hline \multirow{7}{*}{$\begin{array}{l}\text { U } \\
\frac{0}{N} \\
\frac{0}{0} \\
\frac{\pi}{0} \\
\text { U. }\end{array}$} & IG diagnóstico (semanas) & $24,2( \pm 8,5)$ & $25,9( \pm 7,7)$ & 0,124 & & \\
\hline & Diagnóstico $<10 \mathrm{~s}$ & $10(17,2)$ & $63(8,8)$ & 0,034 & $2,159(1,042-4,475)$ & \\
\hline & Jejum alterado & $15(25,9)$ & $148(21)$ & 0,384 & & \\
\hline & Insulina & $28(48,3)$ & $224(31,3)$ & 0,008 & $2,050(1,196-3,513)$ & $1,929(1,115-3,340)$ \\
\hline & IG inicio insulina (semanas) & $28,4( \pm 6,9)$ & $27,7( \pm 6,9)$ & 0,590 & & \\
\hline & Insulina $<10 \mathrm{~s}$ & $2(3,4)$ & $3(0,4)$ & 0,006 & $8,488(1,389-51,853)$ & $5,667(0,905-35,494)$ \\
\hline & $\mathrm{HbA1C}(\mathrm{mmol} / \mathrm{mol})$ & $5,2( \pm 0,61)$ & $5,2( \pm 0,60)$ & 0,566 & & \\
\hline \multirow{3}{*}{$\frac{0}{\frac{t}{\pi}}$} & Cesariana & $24(41,4)$ & $233(32,5)$ & 0,169 & & \\
\hline & Prematuridade & $2(3,4)$ & $50(7)$ & 0,301 & & \\
\hline & IG parto (semanas) & $38,2( \pm 2,0)$ & $38,4( \pm 1,5)$ & 0,448 & & \\
\hline \multirow{5}{*}{$z_{\alpha}$} & Peso nascimento (g) & $3125( \pm 709)$ & $3182( \pm 530)$ & 0,551 & & \\
\hline & Necessidade reanimação & $3(5,2)$ & $19(2,7)$ & 0,314 & & \\
\hline & UCIN & $10(17,2)$ & $38(5,3)$ & $<0,001$ & $3,72(1,743-7,902)$ & \\
\hline & SDR & $4(6,9)$ & $12(1,7)$ & 0,007 & $4,32(1,354-13,91)$ & \\
\hline & Mortalidade & $0(0)$ & $4(0,6)$ & 0,568 & & \\
\hline
\end{tabular}

OR: odds ratio; $\mathrm{OR}_{\mathrm{aj}}$ : odds ratio ajustado

IMC: índice massa corporal; MFIU: morte fetal in útero; Alt: alteração; IG: idade gestacional; s: semana; UCIN: unidade de Cuidados Intensivos Neonatais; SDR: síndroma de dificuldade respiratória 
Tabela 4 - Estado nutricional ao nascer e variáveis a ele associadas

\begin{tabular}{|c|c|c|c|c|c|c|}
\hline \multicolumn{7}{|c|}{ ESTADO NUTRICIONAL } \\
\hline \multicolumn{7}{|c|}{ GRANDE PARA A IDADE GESTACIONAL (excluindo RN LIG) } \\
\hline & & $\underset{n=38}{S i m}$ & $\begin{array}{c}\text { Não } \\
n=650\end{array}$ & $p$ & OR & $\mathrm{OR}_{\mathrm{aj}}$ \\
\hline \multirow{11}{*}{$\sum_{\substack{2 \pi \\
\Sigma}}^{\frac{\pi}{2}}$} & IMC $\left(\mathrm{kg} / \mathrm{m}^{2}\right)$ & $31,1( \pm 4,8)$ & $26,8( \pm 5,3)$ & $<0,001$ & $0,825[-5,926-2,594]$ & \\
\hline & $\mathrm{IMC}>30 \mathrm{~kg} / \mathrm{m}^{2}$ & $20(54,1)$ & $153(23,6)$ & $<0,001$ & $3,791(1,937-7,419)$ & $2,942(1,661-5,955)$ \\
\hline & Gravidez anterior com DG & $10(36,3)$ & $83(12,1)$ & 0,11 & & \\
\hline & Filho anterior macrossómico & $8(21,6)$ & $39(5,9)$ & $<0,001$ & $4,246(1,825-9,878)$ & $4,062(1,619-10,192)$ \\
\hline & Multiparidade & $3(7,9)$ & $11(1,7)$ & 0,004 & $5,057(1,349-18,952)$ & \\
\hline & Alt metabolismo glicose & $4(10,5)$ & $36(4,9)$ & 0,126 & & \\
\hline & Ganho peso excessivo & $23(60,5)$ & $169(23,6)$ & $<0,001$ & $4,968(2,529-9,759)$ & $5,204(2,555-10,601)$ \\
\hline & Ganho inadequado & $1(2,6)$ & $272(37,0)$ & $<0,001$ & $0,046(0,006-0,338)$ & \\
\hline & Ganho peso em kg & $12,6( \pm 5,2)$ & $10,0( \pm 5,3)$ & 0,019 & $1,042[-6,783-2,569]$ & \\
\hline & Patologia na gravidez & $17(44,7)$ & $160(24,2)$ & 0.005 & $2,501(1,291-4,844)$ & $2,380(1,163-4,868)$ \\
\hline & HTA & $5(13,2)$ & $59(8,9)$ & 0,478 & & \\
\hline \multirow{9}{*}{$\frac{N}{\frac{N}{0}}$} & PEC & $1(2,6)$ & $12(1,6)$ & 0,855 & & \\
\hline & Hidrâmnios & $5(13,2)$ & $24(3,3)$ & 0.002 & $4,495(1,613-12,525)$ & \\
\hline & APP & $1(2,6)$ & $28(3,8)$ & 0,710 & & \\
\hline & RPM & $5(13,2)$ & $53(7,2)$ & 0,174 & & \\
\hline & Jejum alterado & $11(28,9)$ & $140(21,0)$ & 0,253 & & \\
\hline & Insulina (S/N) & $20(52,6)$ & $232(31,5)$ & 0,007 & $2,414(1,253-4,649)$ & \\
\hline & Cesariana & $27(71,1)$ & $230(31,3)$ & $<0,001$ & $5,4(2,633-11,074)$ & \\
\hline & Parto intervencionado & $31(81,6)$ & $413(56,1)$ & 0,002 & $3,464(1,506-7,967)$ & \\
\hline & Prematuridade & $4(10,5)$ & $48(6,5)$ & 0,336 & & \\
\hline \multirow{10}{*}{$\underset{\Upsilon}{z}$} & $I G<35 s$ & $2(5,3)$ & $14(1,9)$ & 0,156 & & \\
\hline & Peso nascimento (g) & $4242( \pm 541)$ & $3122( \pm 485)$ & $<0.001$ & $89,634(-1300,72-938,441)$ & \\
\hline & Reanimação & $2(5,3)$ & $20(2,7)$ & 0,358 & & \\
\hline & Asfixia & $1(2,6)$ & $0(0,0)$ & $<0,001$ & $1,027(0,975-1,082)$ & \\
\hline & Ventilação & $1(2,6)$ & $3(0,4)$ & 0,062 & & \\
\hline & UCIN & $4(10,5)$ & $44(6,0)$ & 0,258 & & \\
\hline & Hipoglicémia & $2(5,3)$ & $27(3,7)$ & 0,615 & & \\
\hline & SDR & $2(5,3)$ & $14(1,9)$ & 0,156 & & \\
\hline & Combinada & $11(28,9)$ & $128(17,4)$ & 0,070 & & \\
\hline & Mortalidade & $1(2,6)$ & $3(0,4)$ & 0,062 & & \\
\hline
\end{tabular}

LEVE PARA A IDADE GESTACIONAL (excluindo RN GIG)

\begin{tabular}{|c|c|c|c|c|c|c|}
\hline & & $\begin{array}{c}\text { Sim } \\
n=75\end{array}$ & $\begin{array}{c}\text { Não } \\
n=660\end{array}$ & $p$ & OR & $\mathrm{OR}_{\mathrm{a}}$ \\
\hline \multirow{3}{*}{ ֻั } & Alteração metabolismo glicose & $7(9,2)$ & $33(4,7)$ & 0,094 & & \\
\hline & Diagnóstico $<10 \mathrm{~s}$ & $5(6,6)$ & $68(9,7)$ & 0,370 & & \\
\hline & Jejum alterado & $12(16,0)$ & $151(21,5)$ & 0,265 & & \\
\hline \multirow{10}{*}{$\begin{array}{l}N \\
\frac{N}{0} \\
\frac{0}{2} \\
\frac{\pi}{0} \\
0\end{array}$} & Ganho peso insuficiente & $41(53,9)$ & $232(36,2)$ & $<0.001$ & $2,353(1,459-3,794)$ & $2,433(1,500-3,946)$ \\
\hline & Ganho peso exagerado & $15(20,0)$ & $154(23,6)$ & 0,486 & & \\
\hline & HTA & $12(15,8)$ & $59(8,9)$ & 0,055 & & $1,976(1,003-3,895)$ \\
\hline & PEC & $4(5,3)$ & $12(1,8)$ & 0,051 & & \\
\hline & APP & $2(2,6)$ & $26(3,9)$ & 0,572 & & \\
\hline & RPM & $4(5,3)$ & $54(7,7)$ & 0,437 & & \\
\hline & Cesariana & $28(36,8)$ & $229(32,8)$ & 0,478 & & \\
\hline & Parto distócico & $49(64,5)$ & $395(56,6)$ & 0,187 & & \\
\hline & Prematuridade & $5(6,6)$ & $47(6,7)$ & 0,959 & & \\
\hline & $\mathrm{IG}<35 \mathrm{~s}$ & $3(3,9)$ & $13(1,9)$ & 0,225 & & \\
\hline \multirow{8}{*}{$\underset{\alpha}{z}$} & Peso nascimento (g) & $2467( \pm 372)$ & $3254( \pm 503)$ & $<0,001$ & $0,624(0,335-2,813)$ & \\
\hline & Necessidade reanimação & $2(2,6)$ & $20(2,9)$ & 0,906 & & \\
\hline & UCIN & $4(5,3)$ & $44(6,3)$ & 0,719 & & \\
\hline & SDR & $2(2,6)$ & $14(2,0)$ & 0,717 & & \\
\hline & Hipoglicémia & $3(3,9)$ & $26(3,7)$ & 0,925 & & \\
\hline & Combinada & $13(17,1)$ & $126(18,1)$ & 0,838 & & \\
\hline & Malformação & $6(7,9)$ & $52(7,4)$ & 0,889 & & \\
\hline & Major & $2(33.3)$ & $26(50)$ & 0,439 & & \\
\hline
\end{tabular}

IMC: índice massa corporal; DG: diabetes gestacional; HTA: hipertensão; PEC: pré-eclâmpsia; APP: ameaça de parto prematuro; RPM: rotura de membranas;(S/N): sim / não; IG: idade gestacional; SDR: síndroma de dificuldade respiratória; UCIN: unidade de Cuidados Intensivos Neonatais; OR: odds ratio; ORaj: odds ratio ajustado 
$\mathrm{Na}$ reclassificação materna, cujo resultado só conhecemos em 527 / 774 (68\%) dos casos podemos verificar que apenas uma das anomalias major - agenesia renal unilateral - ocorreu numa mulher em que foi identificada alteração do metabolismo da glicose. Globalmente foram identificadas 40 mães com alteração permanente do metabolismo da glicose (7,9\% das mães estudadas). Estas alterações foram identificadas sobretudo no grupo das mãe com recém-nascidos LIG e GIG e no grupo 2 naqueles que tiveram hipoglicémia.

Na Tabela 5 compara-se a morbilidade do FMD com os restantes recém-nascidos da MBB em igual período (pelos códigos de GDH), verificamos que no global o FMD nascido entre 2008 e 2010 tem menos morbilidade, a prematuridade é significativamente mais baixa, assim com o número de RN com Síndroma de dificuldade respiratória (SDR) e ocorreram menos RN com IA inferior a 7 ao quinto minuto o que resultou em menos internamentos na UCIN. Pelo contrário o número de RN com paralisia do plexo braquial foi significativamente superior no FMD. No grupo 2 (2011 2013) o padrão manteve-se mas de forma não significativa com exceção da paralisia de Erb que não teve expressão no FMD, mas registou-se uma diferença significativa na incidência de anomalias congénitas.

\section{DISCUSSÃO}

Desta análise encontrámos algumas diferenças entre duas coortes temporais de RN (grupo 1 e 2) que podemos atribuir à mudança dos protocolos instituídos na grávida com DG, sobretudo nos anos mais recentes com a implementação de um protocolo que permite o diagnóstico mais precoce, a valorização da glicémia em jejum, a diminuição dos critérios para administração de insulinoterapia e a abordagem mais criteriosa do RN com hiperbilirrubinémia. ${ }^{9,28}$

O PN e a sua relação com a IG é uma medida clínica fundamental na avaliação do RN e é muitas vezes usado para estimar o risco de morbilidade / mortalidade perinatal. As curvas de crescimento de Lubchenco ${ }^{29}$ publicadas em 1963 e amplamente usadas no FMD, sabe-se atualmente não estarem adaptadas à população portuguesa; não separam os RN por sexo e sobrevalorizam os RN macrossómicos. ${ }^{30}$ As curvas de Olsen ${ }^{27}$ utilizadas no registo nacional de DG, são de base populacional mais recentes, analisam grupos multirraciais e dividem os RN por género. Na população de recém-nascidos de mãe diabética, cujo maior paradigma é o RN macrossómico e/ou GIG pela sua associação à morbilidade periparto e neonatal, é fundamental categorizar o RN de acordo com o seu crescimento intrauterino. ${ }^{16,17,20,31}$

O crescimento fetal no filho de mãe diabética está exaustivamente estudado. Existem fatores não modificáveis e modificáveis a condicionar a sua expressão. Nos fatores não modificáveis incluiu-se o terreno genético (embora se saiba que a longo prazo a sua expressão também possa ser alterada), o sexo fetal, a paridade, a idade materna e a estatura; dos modificáveis fazem parte o peso materno / IMC, ganho ponderal na gravidez, a dieta, atividade física, entre outros. ${ }^{32} \mathrm{O} \mathrm{IMC}$ prévio à gravidez foi consistentemente considerado o fator independente mais determinante de macrossomia fetal e parece ser também independente do controlo metabólico materno. . 2,33

O impacto dos novos critérios diagnósticos de DG na

Tabela 5 - Comparação da morbilidade do FMD com os outros RN da MBB (GDH)

\begin{tabular}{|c|c|c|c|c|c|c|c|c|}
\hline \multirow[b]{3}{*}{ Número } & \multicolumn{4}{|c|}{$2008-2010$} & \multicolumn{4}{|c|}{$2011-2013$} \\
\hline & FMD & MBB* $^{*}$ & & & FMD & MBB* $^{*}$ & & \\
\hline & 421 & 8874 & $p$ & OR & 353 & 7563 & $p$ & OR \\
\hline Cesarianas & $\begin{array}{c}129 \\
(30,6)\end{array}$ & $\begin{array}{l}2409 \\
(27,1)\end{array}$ & 0,156 & & $\begin{array}{c}128 \\
(36,3)\end{array}$ & $\begin{array}{l}2068 \\
(27,3)\end{array}$ & $<0,001$ & $\begin{array}{c}1,512 \\
(1,210-1,889)\end{array}$ \\
\hline Prematuridade & $\begin{array}{c}23 \\
(5,5)\end{array}$ & $\begin{array}{c}895 \\
(10,1)\end{array}$ & 0,002 & $\begin{array}{c}0,515 \\
(0,336-0,789)\end{array}$ & $\begin{array}{c}29 \\
(8,2)\end{array}$ & $\begin{array}{c}776 \\
(10,3)\end{array}$ & 0,124 & \\
\hline UCIN & $\begin{array}{c}26 \\
(6,2)\end{array}$ & $\begin{array}{l}797 \\
(9,0)\end{array}$ & 0,048 & $\begin{array}{c}0,667 \\
(0,446-0,999)\end{array}$ & $\begin{array}{c}22 \\
(6,2)\end{array}$ & $\begin{array}{l}690 \\
(9,1)\end{array}$ & 0,063 & \\
\hline Apgar $<7-5^{\circ}$ minuto & $\begin{array}{c}1 \\
(0,2)\end{array}$ & $\begin{array}{c}54 \\
(0,6)\end{array}$ & 0,332 & & $\begin{array}{c}1 \\
(0,3)\end{array}$ & $\begin{array}{c}85 \\
(1,1)\end{array}$ & 0,136 & \\
\hline SDR & $\begin{array}{c}5 \\
(1,2)\end{array}$ & $\begin{array}{l}398 \\
(4,5)\end{array}$ & 0,001 & $\begin{array}{c}0,256 \\
(0,105-0,622)\end{array}$ & $\begin{array}{c}11 \\
(3,1)\end{array}$ & $\begin{array}{l}216 \\
(2,9)\end{array}$ & 0,775 & \\
\hline Hipoglicémia & $\begin{array}{c}6 \\
(1,4)\end{array}$ & $\begin{array}{c}77 \\
(0,89)\end{array}$ & NA & & $\begin{array}{c}23 \\
(6,5)\end{array}$ & $\begin{array}{c}41 \\
(0,81)\end{array}$ & NA & \\
\hline Hiperbilirrubinémia & $\begin{array}{c}46 \\
(10,9)\end{array}$ & $\begin{array}{l}626 \\
(7,1)\end{array}$ & 0,003 & $\begin{array}{c}1,616 \\
(1,177-2,219)\end{array}$ & $\begin{array}{c}45 \\
(12,9)\end{array}$ & $\begin{array}{c}1043 \\
(13,8)\end{array}$ & 0,649 & \\
\hline Fractura clavícula & $\begin{array}{c}1 \\
(0,2)\end{array}$ & $\begin{array}{c}17 \\
(0,2)\end{array}$ & 0,834 & & $\begin{array}{c}1 \\
(0,3)\end{array}$ & $\begin{array}{c}22 \\
(0,3)\end{array}$ & 0,979 & \\
\hline Paralisia Erb & $\begin{array}{c}7 \\
(1,7)\end{array}$ & $\begin{array}{c}9 \\
(0,17)\end{array}$ & $<0,001$ & $\begin{array}{c}16,655 \\
(6,172-44,940)\end{array}$ & 0 & $\begin{array}{c}10 \\
(0,1)\end{array}$ & 0,494 & \\
\hline Malformações & $\begin{array}{c}21 \\
(5,0)\end{array}$ & $\begin{array}{l}446 \\
(5,0)\end{array}$ & 0,972 & & $\begin{array}{c}37 \\
(10,5)\end{array}$ & $\begin{array}{l}257 \\
(3,4)\end{array}$ & $<0,001$ & $\begin{array}{c}3,33 \\
(2,317-4,785)\end{array}$ \\
\hline
\end{tabular}

* RN de mãe sem diabetes; NA: não analisado

FMD: filho de mãe diabética; RN: recém-nascido; MBB: 
saúde materna e neonatal tem sido objeto de análise em várias reuniões nacionais da Sociedade Portuguesa de Diabetologia e também tem sido reportado a diminuição dos recém-nascidos GIG e o aumento dos LIG. Os parâmetros mais frequentemente associados a este achado foram: o IMC prévio à gravidez mais baixo, o ganho ponderal reduzido particularmente nas grávidas com IMC $<25 \mathrm{~kg} / \mathrm{m}^{2}$ (magras ou normais) e a presença de HTA na gravidez. ${ }^{15,34}$ Pudemos objetivar a diminuição da prevalência dos GIG, que se associou a um aumento dos LIG, que passaram a ser mais frequentes. Explorando as razões desta alteração, verificámos que as variáveis fortemente associadas à ocorrência de RN GIG foram a obesidade materna, os antecedentes de DG, de macrossomia fetal e a multiparidade, mas o que parece ter sido determinante foi o aumento ponderal excessivo. Pelo contrário o ganho de peso insuficiente e a existência de complicações obstétricas como a hipertensão foram determinantes para o RN ser LIG. Os fetos GIG e macrossómicos têm um risco aumentado de complicações perinatais relacionadas com o trabalho de parto. ${ }^{2,17,22}$ Ter um feto GIG associou-se a maior necessidade de insulinoterapia e de cesariana mas não se traduziu por aumento da morbilidade neonatal. Um rigoroso controlo metabólico e nutricional materno pode reduzir a incidência de macrossomia / GIG. ${ }^{3,17}$ Reapreciar individualmente a estratégia de orientação da grávida diabética (administração de insulina associada a dieta e exercício) em conjunto com a avaliação ecográfica fetal poderá ser uma forma de controlar o crescimento fetal.

Em regra, o bom controlo metabólico associa-se a uma diminuição da prevalência de hipoglicémia e seria espectável que tal acontecesse sobretudo nos recém-nascidos GIG e LIG ${ }^{35}$ o que não se verificou no nosso trabalho.

Os estudos referem uma taxa de hipoglicémia no FMD de cerca de $24 \%{ }^{36}$ e como é bem conhecido a sua incidência depende do critério de diagnóstico utilizado. Apesar da determinação da glicémia capilar ser menos fiável, sobretudo em situações de hematócrito elevado e glicémia mais baixa, no RN é frequente a sua utilização já que necessita de quantidades reduzidas de sangue e permite o diagnóstico e intervenção. ${ }^{37}$

Na literatura a hipoglicémia no FMD associa-se à obesidade materna, a acidose ao nascer, a ser LIG e à mãe ter necessitado de insulina durante a gravidez e/ou ter tido hiperglicémia durante o trabalho de parto. ${ }^{38}$ Neste trabalho, associou-se sobretudo com a precocidade do diagnóstico e com a morbilidade associada (HTA). Um dos aspectos que não foi explorado foi o do controlo metabólico durante o trabalho de parto. O hiperinsulinismo fetal é o fator etiológico da hipoglicémia neonatal mais bem estudado. No entanto, há um grupo de RNs sem hiperinsulinismo fetal que respondem à hiperglicémia materna durante o trabaIho de parto com a produção excessiva de insulina que vai condicionar hipoglicémia nas primeiras horas de vida. ${ }^{3,39} \mathrm{O}$ protocolo de vigilância destas grávidas durante o trabalho de parto é rigoroso e não se modificou significativamente durante o período em análise.
A diabetes pré-gestacional está associada a um risco elevado de anomalias congénitas, ${ }^{4}$ sendo este risco superior nas mulheres com insuficiente controlo glicémico ${ }^{4} \mathrm{e}$ tem-se mantido estável apesar das estratégias para normalizar a glicémia no momento da embriogénese..$^{17,20,21,40,41} \mathrm{Na}$ população geral, a prevalência de anomalias congénitas é de cerca de 2 a 3\%, ${ }^{17,40,42,43}$ mas nos FMD é ligeiramente superior (1,3 vezes). ${ }^{21,44}$ Este risco está, sobretudo, aumentado nas mulheres com DG que depois se vem a revelar terem diabetes tipo II. 4,20,42 O aumento da prevalência das anomalias congénitas encontrado no nosso trabalho foi uma surpresa e parece estar relacionado com a precocidade do diagnóstico (17,2\% antes das 10 semanas) e necessariamente com o controlo metabólico nesse período tão sensível da gravidez. Não podemos contudo afirmar que este aumento de prevalência seja da responsabilidade da mudança dos critérios de diagnóstico. As anomalias identificadas são as habitualmente descritas nos filhos de grávidas diabéticas, ${ }^{21,40,41}$ registando-se contudo pouca expressão das anomalias do sistema nervoso. Estas anomalias são de fácil identificação na gravidez e as mães poderão ter optado pela interrupção médica da gravidez antes do diagnóstico de DG estar estabelecido.

Na MBB são internados na UCIN cerca de $10 \%$ dos RN, uma taxa superior à dos RN de mãe com DG (6\%). Apesar do aumento da morbilidade documentada a maioria pôde ser vigiado junto da mãe.

A literatura médica sobre os cuidados perinatais à grávida com DG versa sobretudo a otimização dos cuidados pré-natais, procurando melhorar a morbilidade materna, diminuir a macrossomia e assim diminuir as complicações periparto com consequências tão nefastas para o RN.

Os fatores que mais se associaram à morbilidade encontrada que documentámos, foram para a hipoglicémia, a IG do diagnóstico (sobretudo se inferior às 10 semanas) e o jejum alterado e para as anomalias congénitas a necessidade de terapêutica com insulina (principalmente quando necessária antes das 10 semanas). Estes fatores estão ambos associados ao diagnóstico da DG no primeiro trimestre de gravidez (glicémia em jejum como rastreio) e necessidade precoce de insulina, para obtenção de um bom controlo metabólico.

Na reclassificação não registámos um aumento significativo de mulheres com diabetes ou pré-diabetes, mas apenas conhecemos o seu resultado em $68 \%$ das mulheres. Houve no entanto um esforço em orientar as mulheres com diagnóstico mais precoce de DG (primeiro trimestre), mas ao contrário do que seria de esperar, o número de muIheres com alteração permanente do metabolismo da glicose foi menor do que o da amostra global. Desconhecer o seguimento de todas as mulheres é um problema nacional, já que em Portugal só é conhecido o resultado da reclassificação de $68 \%$ das mães ${ }^{15}$

\section{CONCLUSÃO}

Após implementação do novo protocolo de orientação clínico, para diagnóstico e tratamento de DG, registou-se 
um aumento dos casos de hipoglicémia neonatal, de recém-nascidos LIG e de anomalias congénitas. Os fatores que poderão ter contribuído para estas alterações foram as características maternas pré-gravídicas (IMC prévio), o diagnóstico mais precoce, o rigor do controlo metabólico, a sua 'excessiva' adesão às recomendações dietéticas propostas (menor aumento ponderal durante a gravidez) e provavelmente o estado metabólico no momento da embriogénese.

Em conjunto com a equipa de vigilância da grávida diabética, urge desenvolver estratégias para fazer o seguimento de todas as mulheres, mas sobretudo naquelas em que ocorreu morbilidade neonatal significativa.

\section{AGRADECIMENTOS}

A todos os elementos do núcleo de diabetes e gravidez da Maternidade Bissaya Barreto sem os quais este trabalho não teria sido possível, em especial para a Maria Céu Almeida responsável pelo preenchimento da parte obstétrica da base de dados utilizada.

A Margarida Marques do Laboratório de Bioestatística e Informática Médica do Centro Hospitalar e Universitário

\section{REFERÊNCIAS}

1. Watson D, Rowan J, Neale L, Battin M. Admissions to neonatal intensive care unit following pregnancies complicated by gestational or type 2 diabetes. Aust N Z J Obstet Gynaecol. 2003;43:429-32.

2. Mitanchez D, Burguet A, Simeoni U. Infants born to mothers with gestational diabetes mellitus: Mild neonatal effects, a long-term threat to global health. J Pediatr. 2014;164:445-50.

3. NICE. Diabetes in pregnancy: management of diabetes and its complications from preconception to the postnatal period. 2015. [consultado 2016 jul 12]. Disponível em: https://www.nice.org.uk/ guidance/ng3.

4. Dailey TL, Coustan DR. Diabetes in pregnancy. Neoreviews. 2010;11:e619-26.

5. Reece EA. The fetal and maternal consequences of gestational diabetes mellitus. J Matern Fetal Neonatal Med. 2010;23:199-203.

6. Yogev Y, Metzger BE, Hod M. Establishing diagnosis of gestational diabetes mellitus: Impact of the hyperglycemia and adverse pregnancy outcome study. Semin Fetal Neonatal Med. 2009;14:94-100.

7. Simões A, Robalo R, Gomes G, Aleixo F. Diabetes gestacional nos anos 2000 e 2010: retrato de uma sociedade. Rev Port Endocrinol Diabetes Metab. 2013;8:21.

8. Tieu J, McPhee AJ, Crowther CA, Middleton P. Screening and subsequent management for gestational diabetes for improving maternal and infant health. Cochrane Database Syst Rev. 2014;2:CD007222.

9. Sociedade Portuguesa de Endocrinologia Diabetes e Metabolismo, Sociedade Portuguesa de Diabetologia, Sociedade Portuguesa de Obstetrícia e Medicina Materno-Fetal, Seção de Neonatologia da Sociedade Portuguesa de Pediatria. Relatório de Consenso sobre Diabetes e Gravidez. Lisboa: SPEDM, SPD, SPOMMF, SPP; 2011.

10. Ramos G, Moore T. Endocrine disorders in pregnancy. In: Gleason G, Devaskar S, editors. Avery's disease of the newborn. $9^{\text {th }}$ ed. Philadelphia: Elsevier / Saunders; 2012. p. 75-87.

11. Stewart Z, Murphy H. Gestational diabetes. Medicine. 2014;43:44

12. Metzger BE, Persson B, Lowe LP, Dyer AR, Cruickshank JK, Deerochanawong $\mathrm{C}$, et al. Hyperglycemia and adverse pregnancy outcome study: neonatal glycemia. Pediatrics. 2010;126:e1545-52.

13. Reece EA, Moore T. The diagnostic criteria for gestational diabetes: To change or not to change? Am J Obstet Gynecol. 2013;208:255-9.

14. Visser GH, De Valk HW. Is the evidence strong enough to change the diagnostic criteria for gestational diabetes now? Am J Obstet Gynecol. 2013;208:260-4.

15. Massa AC, Rangel R, Cardoso M, Campos A. Diabetes gestacional e o impacto do actual rastreio. Acta Med Port. 2015;28:29-34.

16. Reece EA, Leguizamón G, Wiznitzer A. Gestational diabetes: the need de Coimbra pela preciosa ajuda na realização da análise estatística.

\section{PROTEÇÃO DE PESSOAS E ANIMAIS}

Os autores declaram que os procedimentos seguidos estavam de acordo com os regulamentos estabelecidos pelos responsáveis da Comissão de Investigação Clínica e Ética e de acordo com a Declaração de Helsínquia da Associação Médica Mundial.

\section{CONFIDENCIALIDADE DOS DADOS}

Os autores declaram ter seguido os protocolos do seu centro de trabalho acerca da publicação de dados.

\section{CONFLITOS DE INTERESSE}

Os autores declaram não terem qualquer conflito de interesse relativamente ao presente artigo.

\section{FONTES DE FINANCIAMENTO}

Os autores declaram não ter recebido subsídios ou bolsas para a elaboração do artigo.

for a common ground. Lancet. 2009;373:1789-97.

17. Ramos GA, Hanley AA, Aguayo J, Warshak CR, Kim JH, Moore TR Neonatal chemical hypoglycemia in newborns from pregnancies complicated by type 2 and gestational diabetes mellitus - the importance of neonatal ponderal index. J Matern Fetal Neonatal Med. 2012;25:26771.

18. Hunt KF, Whitelaw BC, Gayle C. Gestational diabetes. Obstet Gynaecol Reprod Med. 2015;24:238-44.

19. Fong A, Serra A, Gabby L, Wing D, Berkowitz K. Hemoglobin A1c as an early predictor of gestational diabetes in obese patients: a subgroup analysis. Am J Obstet Gynecol. 2014;210:S137.

20. Langer O. Pregnancy complicated by diabetes mellitus. In: Martin R, Fanaroff A, Walsh M, editors. Neonatal-perinatal medicine. 9th ed. St Louis: Elsevier; 2011. p. 291.

21. Ogata ES. Problems of the infant of the diabetic mother. Neoreviews. 2010;11:e627-31.

22. Vandorsten JP, Dodson WC, Espeland MA, Grobman WA, Guise JM Mercer BM, et al. NIH consensus development conference: diagnosing gestational diabetes mellitus. NIH Consens State Sci Statements. 2013;29:1-31.

23. Hartling L, Guthrie A, Muise M, Vandermeer B, Donovan L. Benefits and harms of treating gestational diabetes mellitus: a systematic review and meta-analysis for the $U$.S . preventive of medical applications of research. Ann Intern Med. 2013;159:123-9.

24. Correia LG, Boavida JM, Almeida JPF de, Cardoso SM, Dores J, Duarte JS, et al. Diabetes: Factos e Números 2013 - Relatório Anual do Observatório Nacional da Diabetes. Lisboa: Sociedade Portuguesa de Diabetologia; 2013.

25. Brás L, Figueiredo L, Fonseca F. The influence of obesity and gestational weight gain on the newborn weight in a group of women with gestational diabetes. Rev Port Endocrinol Diabetes e Metab. 2013;8:70-6.

26. Institute of Medicine. Weight gain during pregnancy. Inst Med Natl Acad. 2009:1-2.

27. Olsen IE, Groveman SA, Lawson ML, Clark RH, Zemel BS. New intrauterine growth curves based on United States data. Pediatrics. 2010;125:e214-24.

28. Vaz A, Taborda A. Hiperbilirrubinémia - protocolo de actuação da MBB. Coimbra: Maternidade Bissaya Barreto; 2010.

29. Lubchenco LO, Hansman C, Boyd E. Intrauterine growth in length and head circumference as estimated from live births at gestational ages from 26 to 42 weeks. Pediatrics. 1966;37:403-8.

30. Cunha M, Marques A, Carreiro H, Machado M do C. Percentis do peso de nascimento para a idade gestacional, numa população de recém- 
nascidos. Acta Pediátr Port. 2007:38:187-93.

31. Kim SY, Sharma AJ, Sappenfield W, Wilson HG, Salihu HM. Association of maternal body mass index, excessive weight gain, and gestational diabetes mellitus with large-for-gestational-age births. Obstet Gynecol. 2014:123:737-44.

32. Henriksen T. The macrosomic fetus: a challenge in current obstetrics. Acta Obstet Gynecol Scand. 2008;87:134-45.

33. Brás L, Figueiredo L, Fonseca F. The influence of obesity and gestational weight gain on the newborn weight in a group of women with gestational diabetes. Rev Port Endocrinol Diabetes Metab. 2013;8:70-6.

34. Almeida M, Amaral N, Dores J. Diabetes gestacional e o peso ao nascimento - o paradigma invertido? Rev Port Diabetes. 2015;10:3-10.

35. Persson B. Neonatal glucose metabolism in offspring of mothers with varying degrees of hyperglycemia during pregnancy. Semin Fetal Neonatal Med. 2009;14:106-10.

36. Menato G, Bo S, Signorile A, Gallo ML, Cotrino I, Poala CB, et al. Current management gestational diabetes mellitus. Expert Rev Obstet Gynecol. 2008;3:73-91.

37. Flores-le Roux JA, Sagarra E, Benaiges D, Hernandez-Rivas E,
Chillaron JJ, Puig de Dou J, et al. A prospective evaluation of neonatal hypoglycaemia in infants of women with gestational diabetes mellitus. Diabetes Res Clin Pract. 2012;97:217-22.

38. Rozance PJ. Update on neonatal hypoglycemia. Curr Opin Endocrinol Diabetes Obes. 2014:21:45-50.

39. Cowett RM. Neonatal care of the infant of the diabetic mother. Neoreviews. 2002;3:e190-6.

40. Nizard J, Ville Y. The fetus of a diabetic mother: Sonographic evaluation. Semin Fetal Neonatal Med. 2009;14:101-5.

41. Nold JL, Georgieff MK. Infants of diabetic mothers. Pediatric Clinics of North America. 2004;51:619-37.

42. Teramo K. Diabetic pregnancy and fetal consequences. Neoreviews. 2014;15:e83-90.

43. Barthell J, Georgieff M. Infants of diabetic mothers. In: Buonocore G, Bracci RW, editors. Neonatology, a practical approach to neonatal diseases. $1^{\text {st }}$ ed. Milan: Springer-Verlag; 2012. p. 379

44. Lepercq J, Timsit J. Diabètes préalables à la grossesse: complications périnatales. Arch Pediatr. 2005;12:763-5. 